\title{
Lock acquisition of a gravitational-wave interferometer
}

\author{
M. Evans, N. Mavalvala, P. Fritschel, R. Bork, B. Bhawal, R. Gustafson, W. Kells, M. Landry, \\ D. Sigg, R. Weiss, S. Whitcomb, and H. Yamamoto
}

LIGO Hanford Observatory, P.O. Box 1970 S9-02, Richland, Washington 99352

Received September 26, 2001

\begin{abstract}
Interferometric gravitational-wave detectors, such as the Laser Interferometer Gravitational Wave Observatory (LIGO) detectors currently under construction, are based on kilometer-scale Michelson interferometers, with sensitivity that is enhanced by addition of multiple coupled optical resonators. Reducing the relative optic motions to bring the system to the resonant operating point is a significant challenge. We present a new approach to lock acquisition, used to lock a LIGO interferometer, whereby the sensor transformation matrix is dynamically calculated to sequentially bring the cavities into resonance. (C) 2002 Optical Society of America OCIS codes: $120.3180,140.4780,120.2230$.
\end{abstract}

Interferometric gravitational-wave detectors use variants of the Michelson interferometer to convert gravitational-wave strain into optical phase difference in an efficient manner. ${ }^{1}$ Resonant optical cavities are typically used in the arms to build up the interaction time of the light beams with the gravitational wave, increasing the phase difference by a factor proportional to the cavity finesse; the Laser Interferometer Gravitational Wave Observatory (LIGO) interferometers, e.g., contain a 2- or 4-km-long cavity in each arm of the Michelson interferometer. A partially transmitting mirror at the input couples these arm cavities to form a third resonant cavity, the power-recycling cavity, that builds up the power that is incident on the beam splitter. To achieve full sensitivity requires that resonance of each cavity and destructive interference at the antisymmetric port of the interferometer be maintained within a tolerance of $10^{-10}-10^{-13} \mathrm{~m}$, against a continuous background of ground motion driving the optic supports. To reduce these disturbances, all detectors implement an isolation system from which the mirrors are suspended as pendulums; in the LIGO this system provides high isolation for frequencies above $\sim 40 \mathrm{~Hz}$ but minimal isolation below a few hertz.

The LIGO control system has two distinct functions: to maintain the interferometer at the operating point by use of linear time-invariant feedback from a phase-modulation-demodulation system that provides linear error signals for small deviations (of order $1 \mathrm{~nm}$ ) from the operating point ${ }^{2,3}$ and to acquire lock by initially stabilizing the relative optical positions to establish the resonance conditions and bring them within the linear regions of the error signals. Previous prototype interferometers (lengths up to tens of meters) approached lock acquisition in various ways, some as rudimentary as the wait for it to lock approach, in which random ground motion coincidentally positions the mirrors near resonance; more deliberate methods include a relative velocity-damping technique used for a single-arm cavity. ${ }^{4}$ Here we present a new approach to lock acquisition of a power-recycled, Fabry-Perot Michelson interferometer used to lock the LIGO Hanford Observatory's 2-km interferometer. Through a time-domain simulation of the interferometer, an algorithm has been developed that sequentially brings into lock the various degrees of freedom (DOF) by use of measurable optical signals to estimate the time-evolving sensing matrix and application of feedback forces to reduce the mirrors' relative velocities. ${ }^{5}$

The acquisition strategy uses the sensors designed for linear control of the resonant interferometer's four longitudinal DOF (Fig. 1; Refs. 2 and 3). Rf phase-modulation (PM) sidebands are imposed on the input light, and the outputs of photodetectors at three ports are demodulated in both phases at the $\mathrm{rf}$ frequency to yield four linearly independent signals. These signals naturally separate into common and differential mode motions: common and differential arm lengths, $L_{+}$and $L_{-}$, respectively; recycling-cavity length $l_{+}$; and Michelson length $l_{-}$. The nominal lengths are chosen so that at the operating point the first-order PM sidebands (i) resonant in the recycling cavity, (ii) are nearly antiresonant in the arm cavities, and (iii) transmit efficiently to the antisymmetric port (due to a $30-\mathrm{cm}$ difference between $l_{1}$ and $l_{2}$ ).

Initially the suspended optics are damped within their local reference frame; ground motion and the equivalent effect of input-light frequency fluctuations cause the four (real or apparent) lengths to fluctuate by $0.1-1 \mu \mathrm{m} \mathrm{rms}$ over time scales of $0.5-10 \mathrm{~s}$. The probability of all four DOF being simultaneously within the $\sim 1-\mathrm{nm}$ linear region of the resonance

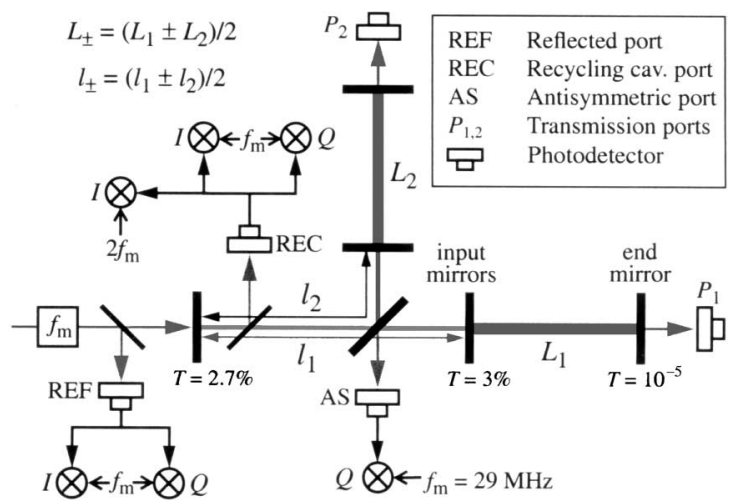

Fig. 1. Power-recycled interferometer with the detection ports shown. In practice, the REC comes from the antireflection-coated surface of the beam splitter, and REF is separated from the input beam with a Faraday isolator. Signals at modulation frequency $f_{m}$ are measured at the REF, REC, and AS by use of in- and quadrature-phase demodulation to generate length error signals (e.g., $I_{\text {ref }}$ and $Q_{\text {ref }}$; the signal at $2 f_{m}$ is measured at the REC. 
points is thus extremely small, and a sequential locking approach must be taken, whereby DOF are captured in sequence (see Fig. 3). First, the recycling cavity and Michelson lengths are brought to the point where the former is resonant for the rf sidebands and the latter is at a carrier dark fringe. In this state, called state 2 since two DOF are locked, the carrier is antiresonant in the recycling cavity, as it does not yet experience the $\pi$ phase shift in reflecting from resonant, overcoupled arm cavities. Next, one of the arm cavities is captured at a carrier resonance; in this state 3 , lengths $l_{+}$and $l_{-}$are unchanged, so the carrier interferes destructively on the symmetric side of the beam splitter, and there is still no significant buildup of the carrier in the recycling cavity. Finally, a carrier resonance of the other arm cavity is captured and full power buildup is achieved in state 4 .

Recovering error signals for each DOF during the acquisition sequence is more of a challenge. The optical gain matrix, $\mathbf{G}$, relating the DOF $\mathbf{D}$ to the output signals $(\mathbf{O}=G \mathbf{D})$, changes throughout acquisition. Our approach capitalizes on the fact that in the LIGO control system the interferometer signals and the control signals are connected with a real-time digital control system capable of significant computation. The basic idea is to estimate the dominant elements of $\mathbf{G}$, continually update the estimation by use of measured power levels in the interferometer, and use the estimate to dynamically construct a sensor transformation matrix $\mathbf{M}$ to form the length error signals from the sensor signals: $\quad \mathbf{D}=M \mathbf{O}$.

The elements of $\mathbf{G}$ are estimated with a generalization of the demodulation signals given in Ref. 3 . They follow the general form $G_{i j}=g_{i j} \sum A_{\mathrm{LO}} A_{\text {res }} \gamma_{\mathrm{sig}}$, relating the $i$ th DOF to the $j$ th detection port. Each signal is the product of a local oscillator field $A_{\mathrm{LO}}$, a resonant field $A_{\text {res }}$ from which signal sidebands are generated, and a factor $\gamma_{\text {sig }}$ representing the optical gain of the signal sidebands; the summation sign reflects the fact that more than one local oscillator or resonant field may contribute to a signal. The scaling factors $g$ contain the fixed optoelectronic gain of the sensing hardware (e.g., the reflectivities of the beam-sampling optics and the photodetector gain). The essential elements of $\mathbf{G}$, given in Table 1, allow us to construct robust error signals for all DOF throughout the acquisition path, with one exception described below. We note that, by scaling the demodulation signals by the field amplitudes, we can account for the effects of alignment fluctuations on the power buildup in the interferometer.

In state 2, both DOF use the carrier for the local oscillator field; we use the reflection port demodulation signals since there is very little carrier in the recycling cavity, and the $2 \times 2$ matrix $M$ is diagonal: $\quad M_{Q_{\mathrm{ref}}, l_{-}}=G_{l_{-}, Q_{\mathrm{ref}}}^{-1}$ and $M_{I_{\mathrm{ref}}, l_{+}}=G_{l_{+}, I_{\mathrm{ref}}}^{-1}$. The $l_{-}$ signal continues to be derived from $Q_{\text {ref }}$ until state 4. In state 3 , the arm-cavity signal appears strongly at the antisymmetric port but is also present in $I_{\text {ref }}$, along with $l_{+}$. We thus invert the $2 \times 2$ matrix $\left(M_{2}\right)$ relating $\left\{I_{\text {ref }}, Q_{\text {as }}\right\}$ to $\left\{l_{+}, L_{+}\right\}$. Entering state 4 , another demodulation signal, $I_{\text {rec }}$, must be added to sense the last DOF. Three of the four DOF are strongly mixed at this point: Until both arms are close to resonance, $L_{ \pm}$are mixed at all detection ports, and $l_{+}$now also appears in the $I_{\text {rec }}$ signal. We thus invert the $3 \times 3$ matrix $\left(M_{3}\right)$ relating $\left\{I_{\text {ref }}, I_{\text {rec }}, Q_{\text {as }}\right\}$ to $\left\{l_{+}, L_{+}, L_{-}\right\}$.

The resulting matrix $\mathbf{M}$ produces robust error signals for the four DOF, with one significant caveat: At some point as the power builds up in the arms, the $I_{\text {rec }}$ and $I_{\text {ref }}$ signals become degenerate, making $l_{+}$and $L_{+}$inseparable. This happens when $A_{\text {Srec }} / A_{\text {Sref }}=A_{C \text { rec }} /\left(A_{C \text { ref }}-A_{S 2 \text { ref }}\right)$, a point that is almost inevitably crossed: The carrier recycling gain $\left(\propto A_{C \text { rec }}\right)$ increases from less than unity in state 3 to some value greater than the sideband recycling gain when full power buildup is reached in state 4 ; at the same time the carrier reflectivity $\left(\propto A_{\text {Cref }}\right)$ decreases from near unity to some small value, typically less than the sideband reflectivity.

When the determinant of the $3 \times 3$ optical gain matrix becomes small, it is not possible to derive independent control signals for $l_{+}$and $L_{+}$. Since $L_{+}$ fluctuates more than $l_{+}$and the system is less tolerant to $L_{+}$deviation from resonance, we control $L_{+}$and leave $l_{+}$uncontrolled while the determinant is below some threshold. In this interval, control signals for $L_{+}$and $L_{-}$are derived from $I_{\text {ref }}$ and $Q_{\text {as }}$ by inversion of the $2 \times 2$ matrix $\left(M_{4}\right)$. As carrier buildup increases and the determinant again exceeds its threshold, the calculation returns to the $3 \times 3$ matrix $\left(M_{3}\right)$. A flow diagram indicating the algorithm for constructing error signals is shown in Fig. 2.

Given expressions for the optical gain elements and a strategy for forming robust error signals, it remains to connect the parameters in the optical gain expressions to measurable quantities. There are seven field amplitudes and three signal gains to infer. They are all estimated with three direct power measurements and one additional demodulated rf signal. The power measurements are those of the light transmitted by each arm cavity and the reflected light. The REC signal, which produces $I_{\text {rec }}$ and $Q_{\text {rec }}$, is also demodulated at twice the modulation frequency; this $2 f_{m}$ demodulation signal is proportional to the recycling-cavity sideband power and is used to estimate $A_{S \text { rec }}$.

Table 1. Elements of the Optical Gain Matrix, $\boldsymbol{G}^{a}$

\begin{tabular}{|c|c|c|c|c|c|}
\hline \multirow[b]{2}{*}{ DOF } & \multicolumn{5}{|c|}{ Demodulation Output } \\
\hline & $I_{\text {ref }}$ & $Q_{\mathrm{ref}}$ & $I_{\text {rec }}$ & $Q_{\text {rec }}$ & $Q_{\text {as }}$ \\
\hline$l_{-}$ & & $\begin{array}{c}\left(A_{\text {Cref }}\right. \\
\left.+A_{S 2 \text { ref }}\right) \\
\times A_{S \text { rec }} \gamma_{\text {prm }}\end{array}$ & & $\begin{array}{l}A_{C \text { ref }} A_{S \text { rec }} \\
\times \gamma_{\text {prm }}\end{array}$ & \\
\hline$l_{+}$ & $\begin{array}{c}\left(A_{C \mathrm{ref}}\right. \\
\left.-A_{S 2 \mathrm{ref}}\right) \\
\times A_{S \mathrm{rec}} \gamma_{\mathrm{prm}}\end{array}$ & & $\begin{array}{c}A_{C \text { rec }} A_{\text {Srec }} \\
\times \gamma_{\text {prm }}\end{array}$ & & \\
\hline $\begin{array}{l}L_{-} \\
L_{+} \\
\end{array}$ & $\begin{array}{l}A_{S \mathrm{ref}} A_{C-} \\
A_{S \mathrm{ref}} A_{C+} \\
\end{array}$ & & $\begin{array}{l}A_{S \mathrm{rec}} A_{C-} \\
A_{S \mathrm{rec}} A_{C+}\end{array}$ & & $\begin{array}{l}A_{S a s} A_{C+} \\
A_{S a s} A_{C-}\end{array}$ \\
\hline
\end{tabular}

${ }^{a}$ In terms of the field amplitudes of the carrier $\left(A_{C}\right)$ and firstand second-order $\mathrm{rf}$ sidebands ( $A_{S}$ and $A_{2 S}$, respectively) at different points in the interferometer. $A_{C}=\gamma_{1} A_{1} \pm \gamma_{2} A_{2}$, where $\left(A_{1}, A_{2}\right)$ are the carrier field amplitudes in the arm cavities. $\gamma$ are optical gain factors for the signal sidebands generated in the arms $\left(\gamma_{1}, \gamma_{2}\right)$ and in the power-recycled Michelson interferometer $\left(\gamma_{\mathrm{prm}}\right)$. 


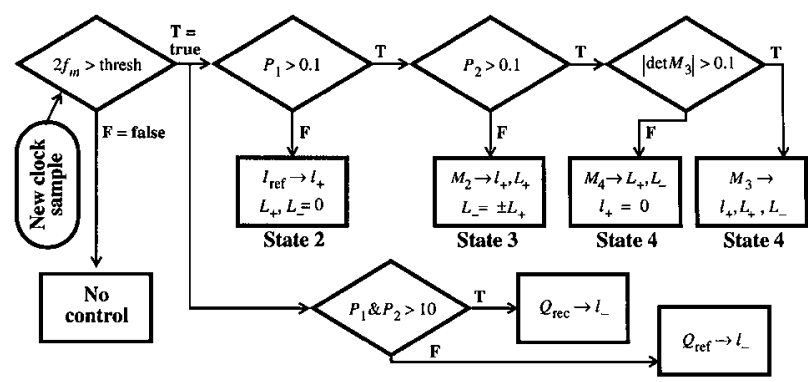

Fig. 2. At each clock sample, five tests (typical values indicated) determine which DOF are controlled from which sensor signals ( $M_{i}$ are defined in the text). Starting from the uncontrolled interferometer, the approach of state 2 is indicated when the $2 f_{m}$ signal crosses $\sim 30 \%$ of its maximum; the signal's sign distinguishes the desired $\mathrm{rf}$ sideband resonance from a carrier resonance. State 3 is approached when either arm nears a carrier resonance, indicated when its transmission $P_{i}$ crosses a fraction of the state 3 level. State 4 is entered when both $P_{i}$ cross a threshold, triggering calculation of $M_{3}$ and activating the $L_{-}$control signal. When the normalized determinant of $M_{3}$ falls below a threshold, the $\left(L_{+}, l_{+}\right)$error signal separation becomes poor, and the $l_{+}$signal is turned off until the determinant again exceeds threshold. Finally, the $l_{-}$signal is switched from $Q_{\text {ref }}$ to $Q_{\text {rec }}$ when both $P_{i}$ exceed $\sim 10 \times$ the state 3 level, since $Q_{\text {rec }}$ becomes more robust-the reflected signal scales with the reflected carrier field, which becomes small and possibly goes through zero.

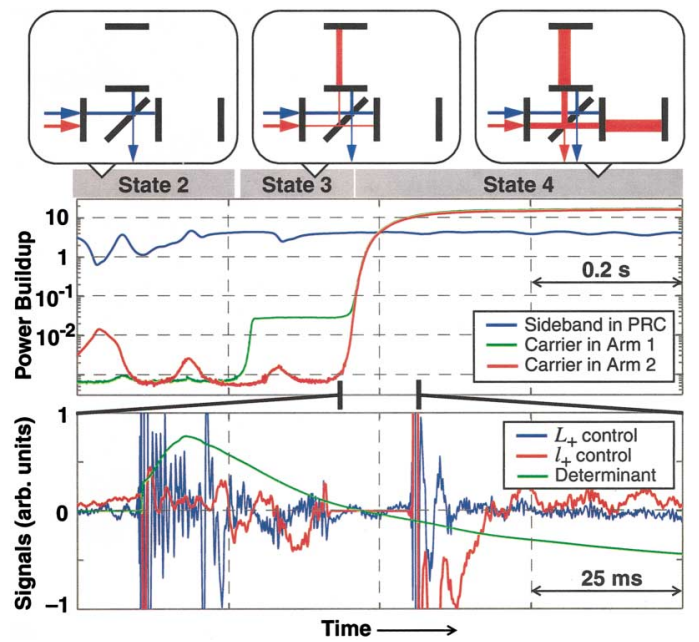

Fig. 3. Top, acquisition states of the interferometer. The rf sidebands (blue) resonate in the power-recycling cavity only, whereas the carrier (red) resonates everywhere. Middle, power levels during acquisition ( $\log _{10}$ vertical scale), normalized to the input sideband power (blue curve) and to the resonant arm power if there were no recycling mirror (green and red curves); shaded bars indicate the corresponding states. Bottom, $L_{+}$and $l_{+}$ control signal and the normalized determinant of $M_{3}$ during the state $3 \rightarrow 4$ transition (central $0.1 \mathrm{~s}$ of upper plot); the determinant goes through zero, and the $l_{+}$ control signal is tuned off for $\sim 15 \mathrm{~ms}$.

Measurements are made in states 2 and 3 to calibrate the signals and to determine the scaling factors $g$.

Feedback control forces derived from these error signals are applied to the suspended optics. The $L_{+}$and
$L_{-}$signals are sent to both end test masses, driven in phase or in antiphase, respectively; $l_{+}$is sent to the recycling mirror and $l_{-}$to the beam splitter and the recycling mirror. Digital filtering applied to each error signal forms stable feedback loops. As power builds up in state 4 , the pole in the optical response to $L_{+}$ moves from that of the single-arm cavity to the coupled-cavity pole frequency (a few hertz) ; $^{3}$ the $L_{+}$loop remains conditionally stable during this process, picking up additional low-frequency gain.

A typical lock-acquisition event is shown in Fig. 3. The channel sampling and calculation rate is $16,384 / \mathrm{s}$. The digital filtering consists of a real zero at $10 \mathrm{~Hz}$ applied to all four error signals to compensate for the pendulum response and an additional real zero at $\sim 100 \mathrm{~Hz}$ applied to the $L_{+}$and $L_{-}$signals to compensate the arm-cavity pole; unity gain frequencies are $\sim 150 \mathrm{~Hz}$ for the $L$ loops and $\sim 30 \mathrm{~Hz}$ for the $l$ loops. The maximum peak force that can be applied to each mirror is $8 \mathrm{mN}$, with a bandwidth of $\sim 1 \mathrm{kHz}$.

On average the system acquires a lock within 1-2 min. This is so in the presence of average relative velocities of $0.2-0.5 \mu \mathrm{m} / \mathrm{s}$ for $l$ and $0.5-1 \mu \mathrm{m} / \mathrm{s}$ for $L$. If an arm cavity passes through a rf sideband resonance in state 2 or 3 , the $\left(l_{+}, l_{-}\right)$error signals become contaminated, and the lock is typically broken, but state 2 is quickly recovered.

In summary, we have implemented an acquisition sequence to lock a LIGO interferometer, achieving a power-recycling gain of up to 25 . The dynamically calculated sensor transformation matrix does not rely on a gain hierarchy for proper signal separation and deals successfully with the optical gain changes, most notably the singularity inherent in the system. The expected recycling gain of 30-50 should be achieved when the full angular alignment system is activated.

We thank our LIGO colleagues for their essential contributions in building the instruments. This work was supported by the National Science Foundation under Cooperative Agreement PHY-9210038. N. Mavalvala, R. Bork, M. Evans, W. Kells, S. Whitcomb, and H. Yamamoto are also with the LIGO Lab, California Institute of Technology, Pasadena, California 91125. P. Fritschel and R. Weiss are also with the LIGO Lab at the Massachusetts Institute of Technology, Cambridge, Massachusetts 02139. M. Evans's e-mail address is mevans@ligo.caltech.edu.

\section{References}

1. B. C. Barish and R. Weiss, Phys. Today 52(10), 44 (1999).

2. M. W. Regehr, F. J. Raab, and S. E. Whitcomb, Opt. Lett. 20, 1507 (1995).

3. P. Fritschel, R. Bork, G. González, N. Mavalvala, D. Ouimette, H. Rong, D. Sigg, and M. Zucker, Appl. Opt. 40, 4988 (2001).

4. J. Camp, L. Sievers, R. Bork, and J. Heefner, Opt. Lett. 20, 2463 (1995).

5. M. Evans, "Lock acquisition in resonant optical interferometers," Ph.D. dissertation (California Institute of Technology, Pasadena, Calif., 2001). 